\title{
ON AN ACCELERATED PROCEDURE OF EXTRAPOLATION
}

\author{
A. YEYIOS \\ Department of Mathematics, \\ University of Ioannina, \\ Ioannina, GREECE \\ (Received January 15, 1980 and in revised form September 29, 1980)
}

ABSTRACT. This paper presents some theoretical results concerning an extrapolation method, based on a completely consistent linear stationary iterative method of first legree, for the numerical solution of the linear system $A u=b$. The main purpose of the paper is to find ranges for the extrapolation parameter, such that the extrapolation method converges independently of whether the original iterative method is convergent or not.

KEY WORDS AND PHRASES. Linear stationary iterative method of first degree, Extrapolation method.

AMS (MOS) SUBJECT CLASSIFICATION (1970) CODES: $65 F 10$

\section{INTRODUCTION.}

For the numerical solution of the linear system of equations

$$
\mathrm{Au}=\mathrm{b},
$$

where $A$ is a given nonsingular real $n \times n$ matrix, $b$ is a given real vector and $\mathrm{u}$ is the solution-vector, which is to be determined, various iterative methods can be applied. Among them, we consider' a completely consistent linear stationary iterative method of first degree (see e.g. [1]) defin by

$$
u^{(m+:)}=G u^{(m)}+k, \quad m=0,1,2, \ldots,
$$

where $G$ is some real matrix, which is called the iteration matrix of the method (1.2), $k$ is some real vector and $u^{(0)}$ is an arbitrary initial approxi- 
mation to the solution $\mathrm{u}$ of (1.1). Moreover, we have

$$
k=(I-G) A^{-1} b \text { and } \operatorname{det}(I-G) \neq 0 \text {. }
$$

In order to accelerate the rates of convergence of methods like (1.2), various procedures and modifications are used. One of them is the extrapolation method based on (1.2). This is defined by

$$
u^{(m+1)}=\omega\left(G u^{(m)}+k\right)+(1-\omega) u^{(m)}, \quad m=0,1,2, \ldots,
$$

where $\omega$ is a real parameter $(\neq 0)$ called the extrapolation parameter. We note here, that the idea of using an extrapolation parameter, $\omega \neq 0$, appeared long ago in the stationary Richardson method [2], based on (1.1), defined by

$$
u^{(m+1)}=u^{(m)}+w\left(A u^{(m)}-b\right)=(I+\omega A) u^{(m)}-\omega b, \quad m=0,1,2, \ldots,
$$

which follows from $(1.4)$ as a special case if $G=I+A$.

For $\omega=1$, method (1.4) coincides with (1.2). The iteration matrix of method $(1.4)$ is

$$
G_{\omega}=\omega G+(1-\omega) I,
$$

where I is the identity matrix of order $n$. Thus, (1.4) takes the form

$$
u^{(m+1)}=G_{\omega^{u}}{ }^{(m)}+k^{\cdot}, \quad m=0,1,2, \ldots,
$$

where $k^{\prime}=\omega k$. Since

$$
\left(I-G_{\omega}\right) A^{-1} b=\omega(I-G) A^{-1} b=\omega k=k^{\prime}
$$

and

$$
\operatorname{det}\left(I-G_{\omega}\right)=\operatorname{det}(\omega(I-G))=\omega^{n} \operatorname{det}(I-G) \neq 0,
$$

it follows that the extrapolation method is completely consistent with the system (1.1).

The problem which now arises is how the parameter $\omega$ must be chosen in order to have $\rho\left(G_{\omega}\right)<\rho(G)$ with $\rho\left(G_{\omega}\right)<1$, where $\rho(G), \rho\left(G_{\omega}\right)$ are the spectral radii of the matrices $G$ and $G_{\omega}$ respectively.

As is known, the problem of finding a theoretical optimum value for $\omega$, say $\omega_{\text {opt }}$, such that

$$
\rho\left(G_{\omega_{\text {opt }}}\right)=\min _{\omega} \rho\left(G_{\omega}\right) \leq \rho(G) \quad \text { and } \quad \rho\left(G_{\omega_{\text {opt }}}\right)<1,
$$

has been solved in some special cases, but not in the general case. It is 
easy to show (see e.g. [3]) that, if the matrix G has real eigenvalues $\mu_{j}$ such that

$$
\mu_{m} \leq \mu_{j} \leq \mu_{M}<1
$$

then $\rho\left(G_{\omega}\right)<1$ iff $0<\omega<2 /\left(1-\mu_{m}\right)$ and the spectral radius $\rho\left(G_{\omega}\right)$ is minimized if we take $\omega=\omega_{\text {opt }}=2 /\left(2-\left(\mu_{m}+\mu_{M}\right)\right)$. Moreover, we have

$$
\rho\left(G_{\omega_{\text {opt }}}\right)=\left(\mu_{M}-\mu_{m}\right) /\left(2-\left(\mu_{M}+\mu_{m}\right)\right)<1 .
$$

Therefore, in this case, the optimum extrapolation method is always convergent, although $\rho(G)$ is not necessarily less than one. It must be noted that (1.7) holds if $G$ is the matrix $B$ of the Jacobi method, corresponding to a positive definite matrix A of the original system. Then, the optimum JOR method [1] converges. We also note, that if $G$ has real eigenvalues such that $1<\mu_{m} \leq \mu_{j} \leq \mu_{M}$, then $\rho\left(G_{\omega}\right)<1$ iff $2 /\left(1-\mu_{M}\right)<\omega<0$. Moreover, $\omega_{\text {opt }}=2 /\left(2-\left(\mu_{m}+\mu_{M}\right)\right)$ and $\rho\left(G_{\omega_{\text {opt }}}\right)=\left(\mu_{M}-\mu_{m}\right) /\left(\mu_{m}+\mu_{M}-2\right)<1$.

In a recent work [4], a geometrical approach of the general case with $\rho(G)<1$ is discussed, where the construction of a capturing circle of the spectrum of $G$ is required.

In the next section we study extrapolation method (1.6) in order to find ranges for $\omega$ in which convergence is achieved in the general case.

\section{CONVERGENCE THEORY.}

First we observe that, if for some norm of $G$ we have $\|G\|<1$, then for $0<\omega<1$ we obtain

$$
\left\|G_{\omega}\right\|=\|\omega G+(1-\omega) I\| \leq \omega\|G\|+1-\omega<\omega+1-\omega=1
$$

that is, the extrapolation method converges. We assume now that,

$$
\mu_{j}=\rho_{j} e^{i \theta}, \quad j=1(1) n,
$$

are the eigenvalues of $G$, where $0 \leq \rho_{m} \leq \rho_{j} \leq \rho_{M}=\rho(G), \quad i=(-1)^{\frac{1}{2}},\left|\mu_{j}\right|=\rho_{j}$ and $\rho_{m}=\min _{j} \rho_{j}, \rho_{M}=\max _{j} \rho_{j}$. In the sequel we omit the subscript $j$ in $\rho_{j}$, $\theta_{j}$ since no confusion can be made; that is, from now on $\rho, \theta$ are used in place of $\rho_{j}, A_{j}$ respectively. Evidently, the eigenvalues of $G_{\omega}$ are given by

$$
\lambda_{j}=\omega \mu_{j}+1-\omega, \quad j=1(1) n \text {. }
$$


Therefore, for the spectral radius of $G_{\omega}$, we have that

$$
\begin{aligned}
\rho\left(G_{\omega}\right) & =\max _{j}\left|\omega \mu_{j}+1-\omega\right| \leq \max _{j}\left\{\left|\omega \mu_{j}\right|+|1-\omega| j=|1-\omega|+|\omega| \underset{j}{\max }\left|\mu_{j}\right|=\right. \\
& =|1-\omega|+|\omega| \rho(G) .
\end{aligned}
$$

We examine now the convergence of method (1.6) in relation to that of method (1.2). We discuss four basic cases, the first two of which are rather trivial.

Case I: $\rho \equiv 0$. Here we assume that $\mu_{j}=0$ for all $j$. Then $\left|\lambda_{j}\right|<1$ iff $0<\omega<2$. It is obvious that the optimum valu of $\omega$, which minimizes $\rho\left(G_{\omega}\right)$, is $\omega_{\text {opt }}=1$, since $\rho\left(G_{1}\right)=0$.

Case II: $\rho \equiv 1$. Now we have that $\left|\mu_{j}\right|=1$ for all $j$. Then, $\left|\lambda_{j}\right|<1$ iff $2 \omega(\omega-1)(1-\cos \theta)<0$. If $\cos \theta \neq 1$, that is, if $\mu_{j} \neq 1$ for all $j$, then for $0<\omega<1$ we have $\rho\left(G_{\omega}\right)<1$. If $\mu_{j}=1$ for some $j$, then method (1.6) does not converge. In the case where $\mu_{j} \neq 1$ for all $j$, we seek the optimum value of $\omega \in(0,1)$ which minimizes $\rho\left(G_{\omega}\right)$. Suppose that

$$
-1 \leq x_{1} \leq x \leq x_{2}<1 \text {, }
$$

where $x=\cos \theta=\operatorname{Re} \mu_{j}$. It is apparent that

$$
\max _{j}\left|\lambda_{j}\right|^{2}=1-2 \omega(1-\omega)\left(1-x_{2}\right)
$$

and

$$
\min _{\omega}\left\{\max _{j}\left|\lambda_{j}\right|\right\}=\left(\min _{\omega}\left[1-2 \omega(1-\omega)\left(1-x_{2}\right)\right]\right)^{\frac{1}{2}} .
$$

By letting $f(\omega)=1-2 \omega(1-\omega)\left(1-x_{2}\right)$, we easily get that $\min _{\omega} f(\omega)=f\left(\frac{1}{2}\right)=\left(1+x_{2}\right) / 2$, which implies that

$$
\min _{\omega} \rho\left(G_{\omega}\right)=\rho\left(G_{\omega}\right)=\rho\left(G_{\frac{1}{2}}\right)=\left(\left(1+\max _{j} \operatorname{Re} \mu_{j}\right) / 2\right)^{\frac{1}{2}} .
$$

Remark If $\max _{j} \operatorname{Re} \mu_{j}=-1$ (which is valid if $\mu_{j}=-1$ for all $j$ ), then we have $\rho\left(G_{\frac{1}{2}}\right)=0$.

Case III: $0<\rho_{\mathrm{m}} \leq \rho \leq \rho_{\mathrm{M}}=\rho(G)<1$.

In this case $\left|\lambda_{j}\right|<1$ iff

$$
\omega^{2}\left(\rho^{2}+1-2 \rho \cos \theta\right)+2 \omega(\rho \cos \theta-1)<0 \text {. }
$$

Assuming first that $\omega<0,(2.5)$ is equivalent to

$$
\omega\left(\rho^{2}+1-2 \rho \cos \theta\right)+2(\rho \cos \theta-1)>0 .
$$


Sirce $\rho^{2}+1-2 \rho \cos \theta \geq \rho^{2}+1-2 \rho=(1-\rho)^{2}>0$ and $1-\rho \cos \theta \geq 1-\rho>0$, from (2.6) we ob$\operatorname{taj}_{n} \omega>2(1-\rho \cos \theta) /\left(\rho^{2}+1-2 \rho \cos \theta\right)>0$, which contradicts to the hypothesis. If non $\omega>0$, then $(2.5)$ is equivalent to

$$
\omega\left(\rho^{2}+1-2 \rho \cos \theta\right)+2(\rho \cos \theta-1)<0,
$$

which gives $0<\omega<2(1-\rho \cos \theta) /\left(\rho^{2}+1-2 \rho \cos \theta\right) \equiv g(\rho, \theta)$ for all $\mu_{j}$, or equivalently

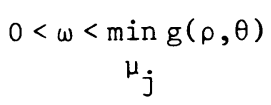

and the following theorem has been proved.

Theorem 2.1 If the method (1.2) converges $(\rho(G)<1)$, then the method $(1.6)$ converges $\left(\rho\left(G_{\omega}\right)<1\right)$ iff $0<\omega<\min _{\mu_{j}}\left\{2(1-\rho \cos \theta) /\left(\rho^{2}+1-2 \rho \cos \theta\right)\right\}$.

Remark It can be proved that $g(\rho, \theta)>1$ for $\rho<1$.

We now show that $\min _{\rho, \theta} g(\rho, \theta)=2 /(1+\rho(G))$ for all $\rho, \theta$ such that

$$
0<\rho_{m} \leq \rho \leq \rho_{M}=\rho(G)<1 \text { and } 0 \leq \theta<2 \pi \text {. }
$$

We note that $\lambda g / \partial(\cos \theta)=\rho\left(1-\rho^{2}\right) /\left(\rho^{2}+1-2 \rho \cos \theta\right)^{2}>0$ from which it follows that

$$
\min g(\rho, \theta)=g(\rho, \cos \theta=-1)=g(\rho, \theta=\pi)=2 /(1+\rho)
$$

and consequently

$$
\min _{\rho, \theta} g(\rho, \theta)=\min _{\rho}(2 /(1+\rho))=2 /\left(1+\rho_{M}\right)=2 /(1+\rho(G)) .
$$

Since, $2 /(1+\rho(G)) \leq \min _{\mu_{j}} g(\rho, \theta)$ (the equality holds if for some $j$ we have $\left.\mu_{j}=-\rho(G)\right)$, then by virtue of Theorem 2.1 the validity of the following two statements is easily established.

Corollary 2.2 If $\rho(G)<1$, then $\rho\left(G_{\omega}\right)<1$ for all $0<\omega<2 /(1+\rho(G))<2$. Corollary 2.3 If the matrix $G$ has at least one negative eigenvalue $\mu$, such that $|\mu|=\rho(G)<1$, then $\rho\left(G_{\omega}\right)<1$ iff $0<\omega<2 /(1+\rho(G))$.

Proof: This follows from Theorem 2.1 since, in this case, we have

$$
\min _{\mu_{j}} g(\rho, \theta)=\min _{\rho, \theta} g(\rho, \theta)=g(\rho(G), \pi)=2 /(1+\rho(G)) \text {. }
$$

Theorem 2.4 If $\rho(G)<1$, then $\rho\left(G_{\omega}\right)<1$ for all $0<\omega<2\left(1-x_{m}\right) /\left(\rho^{2}(G)+1-2 x_{m}\right)$, where $x_{m}=\min x, \quad x=\operatorname{Re} \mu_{j}=\rho \cos \theta$.

Proof: According to Theorem 2.1, it is sufficient to show that 


$$
2 /(1+\rho(G)) \leq 2\left(1-x_{m}\right) /\left(\rho^{2}(G)+1-2 x_{m}\right) \leq \min _{\mu_{j}} g(\rho, \theta) .
$$

Evidently, we have $-1<x_{m} \leq x<1$ and $-\rho(G) \leq x_{m} \leq \rho(G)$. Hence, $\rho^{2}(G)+1-2 x_{m}$ $\geq \rho^{2}(G)+1-2 \rho(G)=(1-\rho(G))^{2}>0$. Thus, the first inequality in (2.8) is equivalent to

$$
\left(\rho(G)+x_{m}\right)(\rho(G)-1) \leq 0
$$

Since $1-\rho(G)>0$ and $\rho(G)+x_{m} \geq 0$, we conclude that (2.9) holds. To prove the second inequality in $(2.8)$, it is sufficient to show that $2\left(1-x_{m}\right) /\left(\rho^{2}(G)+1-\right.$ $\left.-2 x_{m}\right) \leq 2(1-\rho \cos \theta) /\left(\rho^{2}+1-2 \rho \cos \theta\right)$ for $a 11 \mu_{j}=\rho e^{i \theta}$, or equivalently, since $\rho \cos \theta=x$, that

$$
\rho^{2}\left(1-x_{m}\right)-\rho^{2}(G)(1-x)+x_{m}-x \leq 0 .
$$

The inequality $(2.10)$ can be written as follows

$$
(1-x)\left(1-\rho^{2}(G)\right) \leq\left(1-x_{m}\right)\left(1-\rho^{2}\right) \text {. }
$$

The inequality above holds because of the relationships $0<1-\mathrm{x} \leq 1-\mathrm{x}_{\mathrm{m}}$ and $0<1-\rho^{2}(G) \leq 1-\rho^{2}$.

Corollary 2.5 If the iteration matrix G of the method (1.2), has eigenvalues $\mu_{j}$ such that, $\operatorname{Re} \mu_{j} \geq 0$ for all $j$ and $\rho(G)<1$, then $\rho\left(G_{\omega}\right)<1$ for all $0<\omega<2 /\left(1+\rho^{2}(G)\right)$.

Case IV: $0 \leq \rho_{m} \leq \rho \leq \rho_{M}$ and $\rho_{m}<1<\rho_{M}=\rho(G)$.

It is also assumed, in this case, that $\mu_{j} \neq 1, j=1(1) n$. Consider now only those eigenvalues $\mu_{j}$ of $G$ with $1<\rho \leq \rho_{M}=\rho(G)$. Then $\left|\lambda_{j}\right|<1$ iff

$$
\omega^{2}\left(\rho^{2}+1-2 \rho \cos \theta\right)+2 \omega(\rho \cos \theta-1)<0 \text {. }
$$

We distinguish two cases according to whether $\omega$ is less or greater than zero. 1. Let $\omega<0$. Since $\rho^{2}+1-2 \rho \cos \theta \geq(1-\rho)^{2}>0$, by virtue of $(2.11)$ we have $\omega>2(1-\rho \cos \theta) /\left(\rho^{2}+1-2 \rho \cos \theta\right)$. In order that negative values for $\omega$ exist, we must have 1-p $\cos \theta<0$; Namely, the real parts of the eigenvalues, which we consider, must be $>1$. Since, according to cases I, II, and III for the other eigenvalues of $G$ with $0 \leq \rho_{m} \leq \rho<1$, there is no convergence for $w<0$, we conclude that $\omega$ can not take negative values.

2. Now let $\omega>0$. Then (2.11) gives $\omega<2(1-\rho \cos \theta) /\left(\rho^{2}+1-2 \rho \cos \theta\right)$. In order that positive values for $\omega$ exist, we must have $\rho \cos \theta<1$ or $\operatorname{Re} \mu_{j}<1$ for tho- 
se eigenvalues $\mu_{j}$ with $\rho>1$. Considering now all the eigenvalues of $G$ and taking into account the results of Cases I, II, and III, together with the observation that $2(1-\rho \cos \theta) /\left(\rho^{2}+1-2 \rho \cos \theta\right) \leq 1$ for $\rho \geq 1$, the theorem below follows.

Theorem 2.6 If $\rho(G)>1$ and $\operatorname{Re} \mu_{j}<1, j=1(1) n$, then $\rho\left(G_{\omega}\right)<1$ iff $0<\omega$ $<\min g(\rho, \theta)$, where $g(\rho, \theta)=2(1-\rho \cos \theta) /\left(\rho^{2}+1-2 \rho \cos \theta\right)$.

$\mu_{j}$

Theorem 2.7 If $\rho(G)>1$ and $\operatorname{Re} \mu_{j} \leq 0$, then $\rho\left(G_{\omega}\right)<1$ for all $0<\omega<2 /\left(1+\rho^{2}(G)\right)$. Proof: We prove that

$$
2 /\left(1+\rho^{2}(G)\right) \leq 2(1-\rho \cos \theta) /\left(\rho^{2}+1-2 \rho \cos \theta\right) \text { for all } \mu_{j} \text {. }
$$

For this, because of the remark in Theorem 2.1, it is sufficient to show (2.12) for $\rho>1$ and $\rho \cos \theta \leq 0$. We observe that (2.12) is equivalent to

$$
\rho^{2}-1 \leq(1-\rho \cos \theta)\left(\rho^{2}(G)-1\right) \text {, }
$$

which holds because of $0<\rho^{2}-1 \leq \rho^{2}(G)-1$ and $1 \leq 1-\rho \cos \theta$.

The following Theorem is an immediate result of Case IV.

Theorem 2.8 If $G$ has eigenvalues $\mu_{j}$ with $\rho>1, j=1(1) n$, then the following statements are valid.

1. If $\operatorname{Re} \mu_{j}>1, j=1(1) n$, then $\rho\left(G_{\omega}\right)<1$ iff $\max g(\rho, 0)<\omega<0$. $\mu_{j}$

2. If $\operatorname{Re} \mu_{j}<1, j=1(1) n$, then $\rho\left(G_{\omega}\right)<1$ iff $0<\omega<\min g(\rho, \theta)$, where $g(\rho, \theta)=2(1-\rho \cos \theta) /\left(\rho^{2}+1-2 \rho \cos \theta\right)$.

Corollary 2.9 If $G$ has eigenvalues $\mu_{j}$ with $\operatorname{Re} \mu_{j}>1, j=1(1) n$, then $\rho\left(G_{\omega}\right)<1$ for all $2 /(1-\rho(G))<\omega<0$.

Proof: The conclusion follows, in view of the Theorem 2.8, since we have $2(1-\rho \cos \theta) /\left(\rho^{2}+1-2 \rho \cos \theta\right) \leq 2 /(1-\rho(G))$ for all $\mu_{j}$; that is, $\max g(\rho, \theta) \leq 2 /(1-\rho(G))$.

$\mu_{j}$

If the method (1.2) is the Jacobi method ( $\mathrm{J}$ ) with iteration matrix B, then (1.6) is the extrapolation Jacobi method (JOR or EJ) with iteration matrix $B_{\omega}$ (see e.g. [1]). For these methods, by applying the previous results, we have.

Theorem 2.10 If all the eigenvalues of $\mathrm{B}$ are real with moduli $>1$, then the JOR method does not converge. 
Proof: Since $\operatorname{tr}(B)=0$, the matrix $B$ must have positive and negative eigenvalues with moduli $>1$. The proof now follows by theorem 2.8 .

Theorem 2.11 If the Jacobi method (J) converges $(\rho(B)<1)$, then $\rho\left(B_{\omega}\right)<1$ for all $0<\omega<2 /(1+\rho(B))<2$.

The previous Theorem is more general than Theorem $1.1[1, \mathrm{p} .107]$. We also note that some of the results of this section are given in [5] in a different way.

In the sequel, we consider the case where the matrix $G$ of the method (1.2) has eigenvalues $\mu_{j}$ with $0 \neq \rho(G)<1$ and we seek to find values for $w$ such that $\rho\left(G_{\omega}\right) \leq \rho(G)$.

For this, we require $\left|\lambda_{j}\right| \leq\left|\mu_{j}\right|$ to hold for all $j$, where $\lambda_{j}$ are the eigenvalues of $G_{\omega}$, given by (2.2). Because of (2.1), we conclude that $\left|\lambda_{j}\right| \leq\left|\mu_{j}\right|$ iff

$$
F(\omega)=\omega^{2}\left(\rho^{2}+1-2 \rho \cos \theta\right)+2 \omega(\rho \cos \theta-1)+1-\rho^{2} \leq 0 .
$$

Since, $\rho^{2}+1-2 \rho \cos \theta \geq(1-\rho)^{2}>0$ and the discriminant of $F(\omega)$ given by $D=4 \rho^{2}(\rho \cos \theta)^{2} \geq 0$, it follows that (2.13) holds iff $\min \left(\omega_{1}, \omega_{2}\right) \leq \omega \leq \max \left(\omega_{1}, \omega_{2}\right)$, where $\omega_{1}, \omega_{2}$ are the two roots of $F(\omega)$ given by

$$
\omega_{1}=1, \quad \omega_{2}=\left(1-\rho^{2}\right) /\left(\rho^{2}+1-2 \rho \cos \theta\right) .
$$

We note that $\omega_{2}>1$ if $\rho^{2}<\rho \cos \theta$, while $\omega_{2}<1$ if $\rho^{2}>\rho \cos \theta$.

Thus, the following Theorem has been proved.

Theorem 2.12 If $G$ has eigenvalues $\mu_{j}$ with $0 \neq \rho(G)<1$, then the following statements are valid.

1. If $\rho^{2}<\operatorname{Re} \mu_{j}$ for all $j$, then $\rho\left(G_{\omega}\right) \leq \rho(G)$ for all

$$
1 \leq \omega \leq \min \left\{\left(1-\rho^{2}\right) /\left(\rho^{2}+1-2 R e \mu_{j}\right)\right\} \text {. }
$$

2. If $\rho^{2}>\operatorname{Re} \mu_{j}$ for all $j$, then $\rho\left(G_{\omega}\right) \leq \rho(G)$ for all

$$
\max _{j}\left\{\left(1-\rho^{2}\right) /\left(\rho^{2}+1-2 \operatorname{Re} \mu_{j}\right)\right\} \leq \omega \leq 1 .
$$

Remark The restriction $\rho^{2}<\operatorname{Re} \mu_{j}$ implies that $\rho<1$ and thus $\rho(G)<1$, while this is not true if $\rho^{2}>\operatorname{Re} \mu_{j}$.

Letting $\mu_{j}=x+i y$, where $x=\operatorname{Re} \mu_{j}$ and $y=\operatorname{Im} \mu_{j}$, we have $\rho^{2}=x^{2}+y^{2}$. Then the following lemma, which can be easily shown, gives ranges for $x$ and $y$ such that either $\rho^{2}<\mathrm{x}$ or $\rho^{2}>\mathrm{x}$ and $\rho<1$. 
Lemma 2.13

1. $\rho^{2}<x$

iff $|y|<1 / 2$ and $(1-k) / 2<x<(1+k) / 2$, where $k=\left(1-4 y^{2}\right)^{\frac{1}{2}}$.

2. $\rho^{2}>x$ and $\rho<1$ iff anyone of the following statements holds

i) $-1<x<0$ and $|y|<\left(1-x^{2}\right)^{\frac{1}{2}}$

ii) $-1<y<-1 / 2$ or $1 / 2 \leq y<1$ and $0<x<\left(1-y^{2}\right)^{\frac{1}{2}}$

iii) $|y|<1 / 2$ and $(1+k) / 2<x<\left(1-y^{2}\right)^{\frac{1}{2}}$ or $0<x<(1-k) / 2$, where $k=\left(1-4 y^{2}\right)^{\frac{1}{2}}$. As an application of Theorem 2.12, we consider the following examples.

1. Let the matrix $\mathrm{G}$ have eigenvalues

$$
\mu_{1}=\frac{2}{3}+\frac{1}{4} i, \quad \mu_{2}=\frac{2}{3}-\frac{1}{4} i, \quad \mu_{3}=\frac{1}{2}+\frac{1}{5} i, \quad \mu_{4}=\frac{1}{2}-\frac{1}{5} i
$$

satisfying $\rho^{2}<\operatorname{Re} \mu_{j}, \quad j=1,2,3,4$.

Therefore

$$
\rho\left(G_{\omega}\right)<\rho(G) \text { for all } \omega \in\left(1, \min \left\{\left(1-\rho^{2}\right) /\left(\rho^{2}+1-2 \operatorname{Re} \mu_{j}\right)\right\}\right) \equiv(1,2.4483) .
$$

It must be noted that $\rho(G) \approx 0.7120$, while

$$
\min \rho\left(G_{\omega}\right)=\rho\left(G_{1.92}\right)=0.6 \text {. }
$$
$\omega \in(1,2.4483)$

2. Suppose that $G$ has eigenvalues

$$
\mu_{1}=-\frac{2}{3}, \mu_{2}=-\frac{1}{2}+\frac{3}{4} i, \quad \mu_{3}=-\frac{1}{2}-\frac{3}{4} i
$$

satisfying $\rho^{2}>\operatorname{Re} \mu_{j}, \rho<1, j=1,2,3$.

Hence, we have

$$
\left.\rho\left(G_{\omega}\right)<\rho(G) \text { for all } \omega \in \max _{j}\left\{\left(1-\rho^{2}\right) /\left(\rho^{2}+1-2 \operatorname{Re} \mu_{j}\right)\right\}, 1\right) \equiv(0.2,1) \text {. }
$$

Moreover, we find that $\rho(G) \approx 0.9014$ and

$$
\min _{\omega \in(0.2,1)}\left(G_{\omega}\right)=\rho\left(G_{24 / 45}\right) \approx 0.4472
$$

\section{CONCLUDING REMARKS}

From the previous convergence results of section 2 , it is clear, that in onder to be possible to find ranges for $\omega$ such that $\rho\left(G_{\omega}\right)<1$, either $\operatorname{Re} \mu_{j}<1$ or $\operatorname{Re} \mu_{j}>1$ must hold for all the eigenvalues $\mu_{j}, j=1(1)$ n of $G$. For practical purposes and in a case which is not a special one, the choice 
of $\omega_{\text {opt }}$ is made computationally, since, the range for $\omega$ for which $\rho\left(G_{\omega}\right)<1$ is known.

Finally, we note that the Accelerated Overrelaxation (AOR) method studied in [6], which in turn was an extension of the corresponding one introduced by Hadjidimos [7], is an extrapolation of an obvious extension of the well known Successive Overrelaxation method (SOR) (see e.g. [8], [1]). Also, in a paper of Niethammer [9], an extrapolation of the SOR is studied. Thus, all the theory developed in this paper could be applied to the extrapolation method based on SOR in order to obtain better rates of convergence.

ACKNOWLEDGEIENT: The author wishes to thank the referee for his constructive comments and suggestions.

\section{REFERENCES}

1. YOUNG, D.M. Iterative Solution of Large Linear Systems, Academic Press, New York, (1971).

2. RICHARDSON, L.F. The Approximate Arithmetical Solution by Finite Differences of Physical Problems Involving Differential Equations with an Application to the Stresses in a Masonry Dam, Philos. Trans. Roy. Soc. London, Ser. A210 (1910) 307-357.

3. ISAACSON E. and KELLER, H.B. Analysis of Numerical Methods, John Wiley \& Sons, Inc., New York, (1966).

4. de PILLIS, J. and NEUMANN,M. Iterative Methods with k-part splittings, IMA J. of Num. Analysis (submitted).

5. HADJIDIMOS, A. and YEYIOS, A. The Principle of Extrapolation in Connection with the Accelerated Overrelaxation (AOR) Method, Linear Algebra Appl. 30 (1980) 115-128.

6. YEYIOS, A. On the Accelerated Overrelaxation (AOR) Method for Solving Large Linear Systems (Greek), Doctoral Thesis, University of Ioannina, Ioannina, Greece, (1979).

7. HADJIDIMOS, A. Accelerated Overrelaxation Method, Math. Comp. 32 (1978) 149-157.

8. VARGA, R.S. Matrix Iterative Analysis, Prentice-Hall, New Jersey,(1962).

9. NIETHAMMER, W. On Different Splittingsand the Associated Iteration Methods, SIAM J. Numer. Anal. 16 (1979) 186-200. 


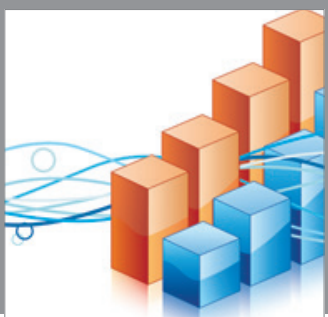

Advances in

Operations Research

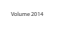

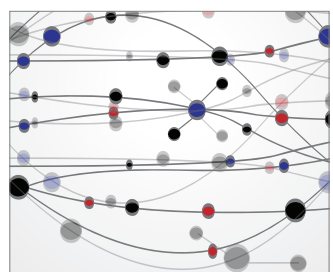

\section{The Scientific} World Journal
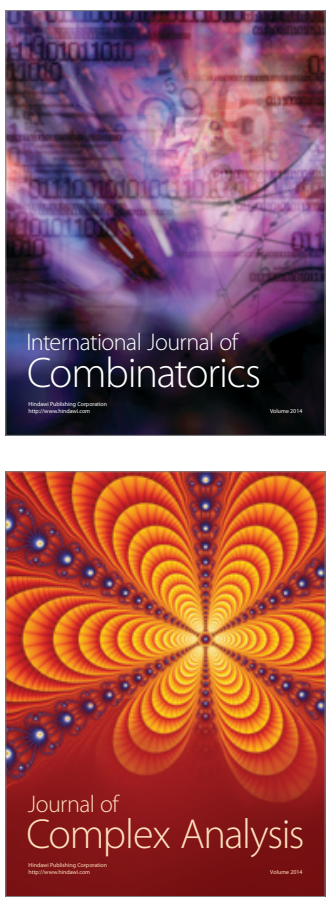

International Journal of

Mathematics and

Mathematical

Sciences
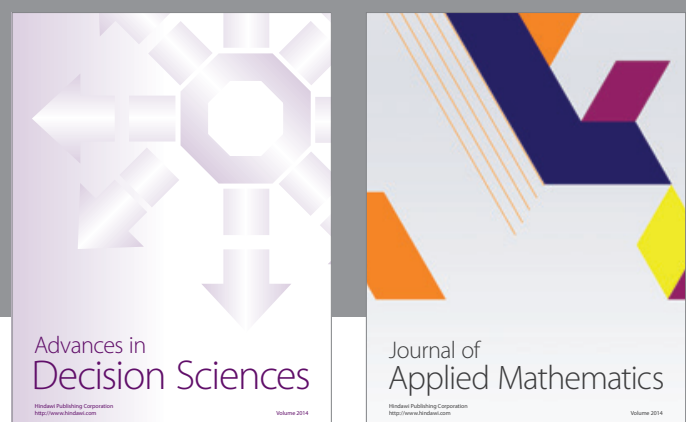

Journal of

Applied Mathematics
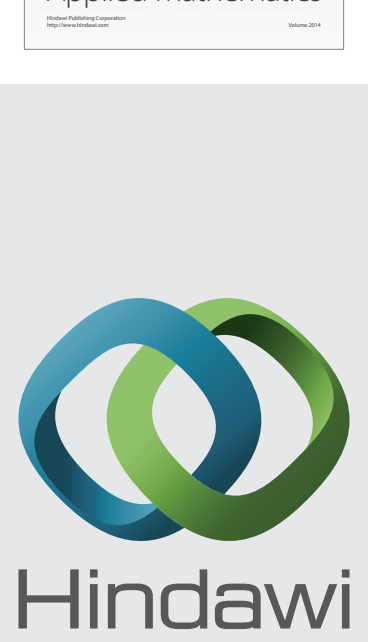

Submit your manuscripts at http://www.hindawi.com
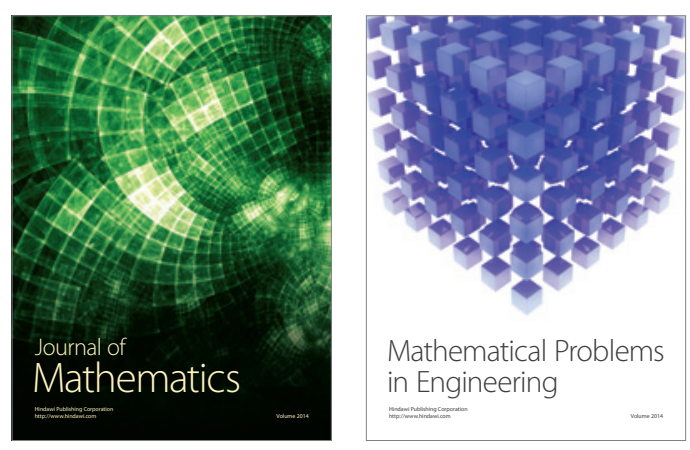

Mathematical Problems in Engineering
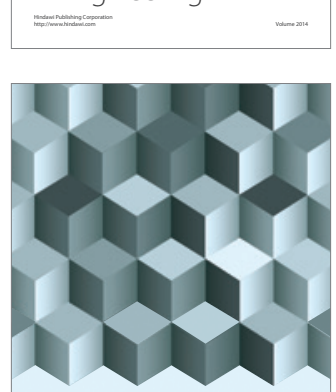

Journal of

Function Spaces
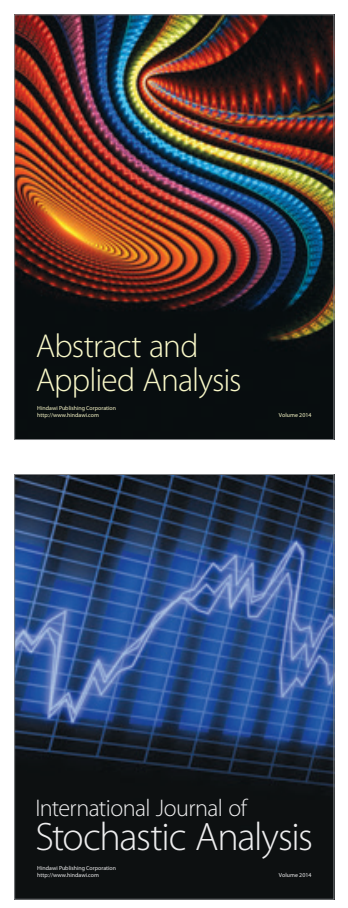

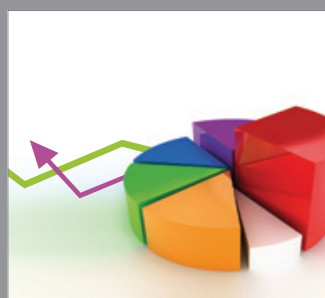

ournal of

Probability and Statistics

Promensencen
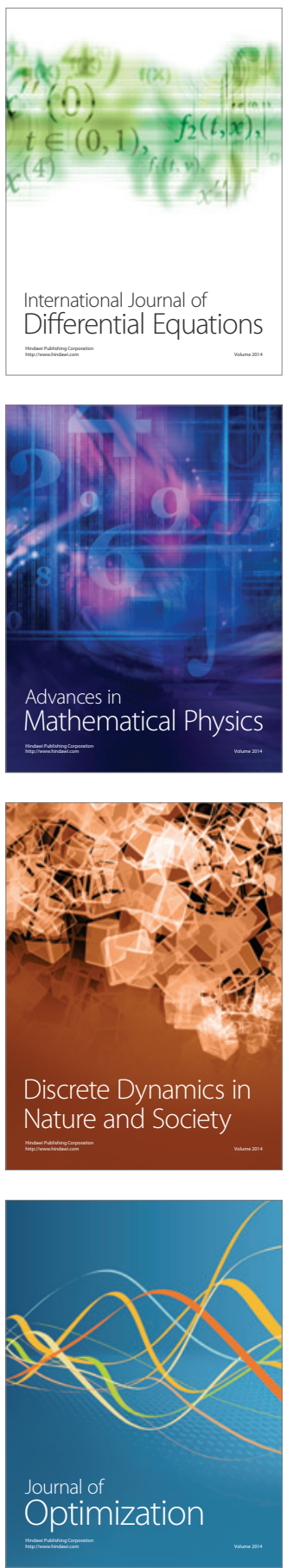